ELECTRONIC SUPPORTING INFORMATION

Ост. 28,2020

\title{
Compressed-State Multistate Pair-Density Functional Theory
}

Jie J. Bao, Chen Zhou, and Donald G. Truhlar

\section{TABLE OF CONTENTS}

Rotation Angles Generating XMS and CMS Intermediate States $\quad$ S-2

$\begin{array}{ll}\text { Figure S1 S-2 } & \end{array}$

$\begin{array}{ll}\text { Figure S2 } & \text { S-2 }\end{array}$

$\begin{array}{ll}\text { Sample Input Files } & \text { S-3 }\end{array}$

$\begin{array}{ll}\text { Absolute Energies } & \text { S-5 }\end{array}$

$\begin{array}{ll}\text { Tables S1-S5 S-5 } & \text { S }\end{array}$ 


\section{Rotation Angles Generating XMS and CMS Intermediate States}

This section compares the XMS rotation angles and the CMS rotation angles for isocyanic acid and the spiro cation. The former are used for XMS-CASPT2 and XMS-PDFT calculations; the latter are used for CMSPDFT calculations. These rotation angles are defined as the angles that change the reference states into the intermediate states. The rotation angles are converted to cosine functions of the angles so that the square of these quantities also mean the weight of the ground reference state in a ground intermediate state.

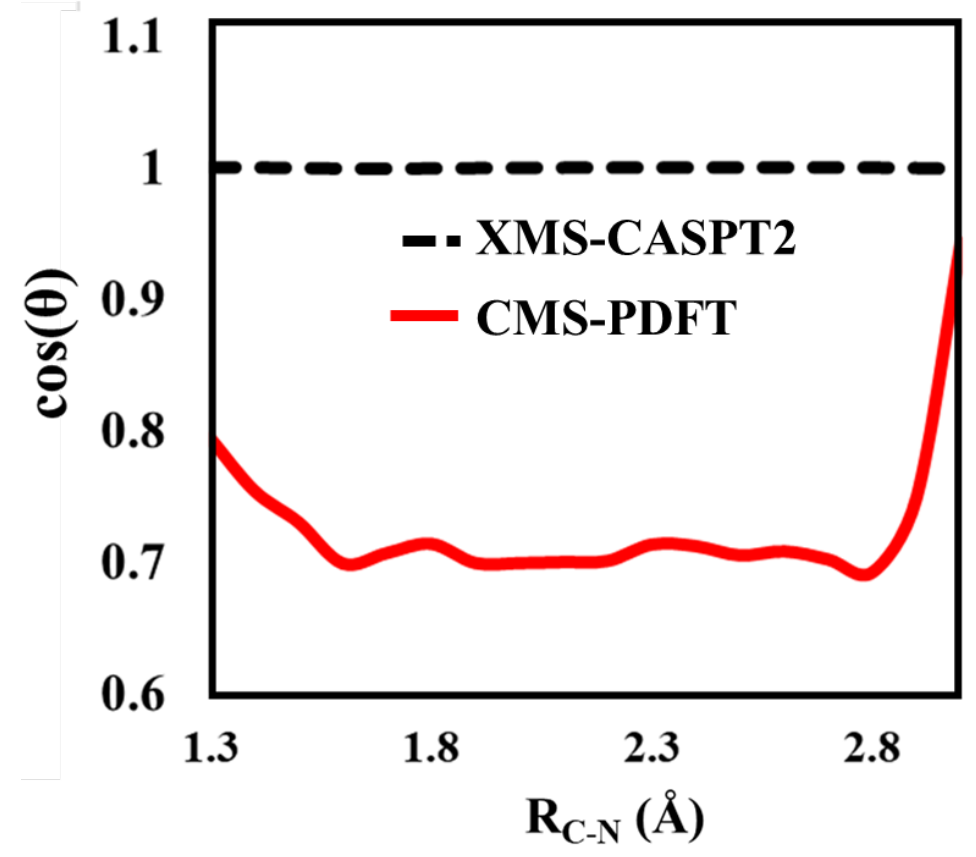

Figure S1. The cosines of the rotation angle that generate the intermediate states for CMS-PDFT (red solid curve) and XMS-CASPT2 (black dashed curve) for isocyanic acid when the dihedral angle is $175 \mathrm{deg}$.

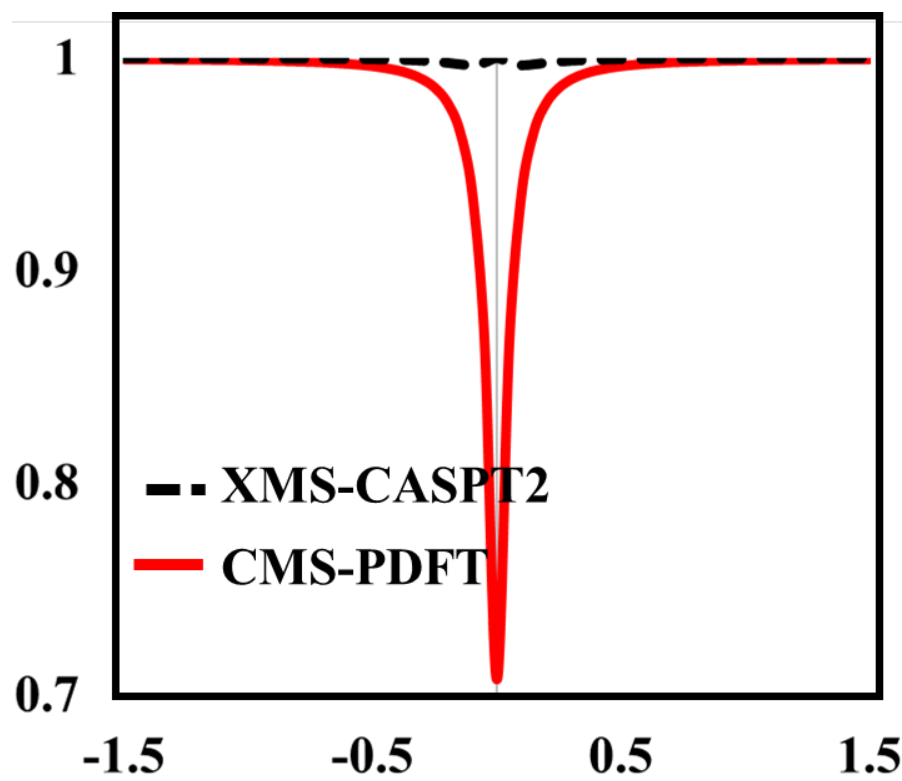

Figure S2. The cosines of the rotation angles that generate the intermediate states for CMS-PDFT (red solid curve) and XMS-CASPT2 (black dashed curve) for the spiro cation. 
Sample Input Files for Performing CMS-PDFT Calculations

Below is a sample input file for performing CMS-PDFT calculations for LiF.

\&GATEWAY

Coord $=$ LiF.xyz

Basis=jun-cc-pVQZ

Group $=$ XY Y

EndofInput

\&SEWARD

EndofInput

\&RASSCF

Spin $=1$

Symmetry $=1$

CIRoot $=221$

Inactive $=2 \quad 0000$

Ras2=3 101

Nactel $=8 \quad 00$

>> C COPY \$CurrDir/LiF.RasOrb \$Scratch/INPORB

\&RASSCF

Lumorb

CIOnly

Spin=1

Symmetry $=1$

CIRoot $=221$

Inactive $=2 \quad 0000$

Ras2=3 101

Nactel $=800$

CMSI

\&MCPDFT

$\mathrm{KSDFT}=\mathrm{TPBE}$

NoGrad

MSPD 
Below is a sample input file for performing CMS-PDFT calculations for the spiro cation

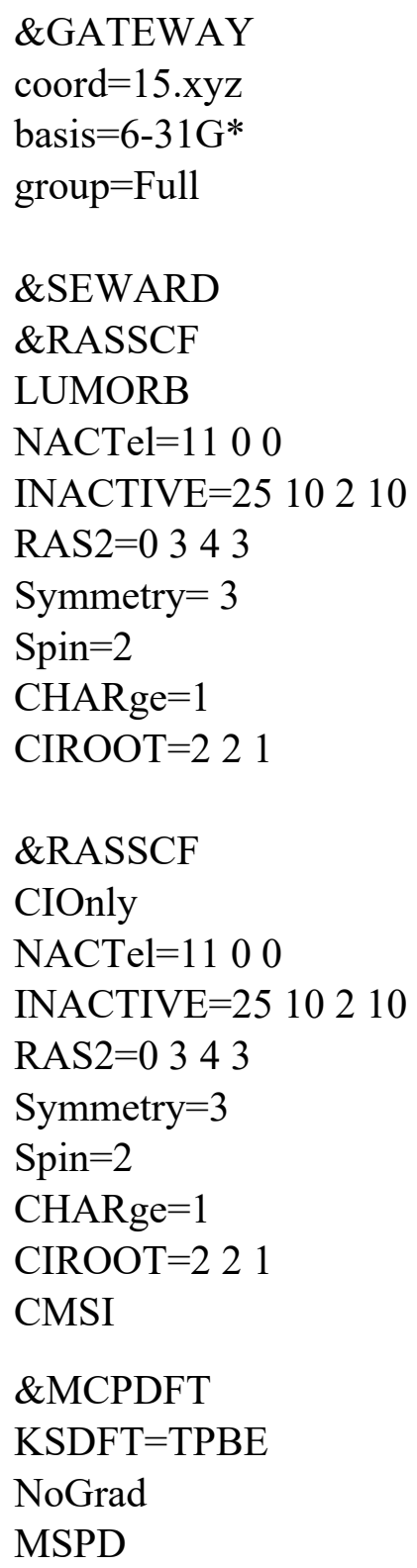




\section{Absolute Energies}

This section lists the CMS-PDFT absolute energies in hartrees for systems that are studied in the paper. The absolute energies of XMS-CASPT2, XMS-PDFT and FMS-PDFT are found in the supplementary information our previous paper. ${ }^{1}$ Distances are in $\AA$.

Table S1. Absolute energies (hartrees) for LiF

\begin{tabular}{|c|c|c|c|c|c|c|}
\hline $\mathrm{Li}-$ & MS-PDFT_1 & CMS-PDFT_2 & XMS-PDFT_1 & XMS-PDFT_2 & $\begin{array}{c}\text { XMS- } \\
\text { CASPT2_1 }\end{array}$ & $\begin{array}{c}\text { XMS- } \\
\text { CASPT2_2 }\end{array}$ \\
\hline 1 & 107.00218985 & -106.75306888 & -107.00372458 & -106.75370389 & 106.98371802 & ra \\
\hline 1.2 & 107.21882493 & -106.94844702 & -107.21897133 & -106.94842268 & -107.21316271 & -1069395770 \\
\hline 1.4 & 107.28661162 & -107.02428522 & -107.28658447 & -107.02448664 & 6235 & 107.0199385 \\
\hline 1.6 & -107.29775246 & -107.05370804 & 107.29797201 & 38 & -107.2 & -107.0511662 \\
\hline 1.8 & -107.28783996 & -107.06 & -107.2 & & -107.2 & -107. \\
\hline 2 & -107.27064979 & -107.07042266 & -107.2 & & -107.27212201 & -107.0704344 \\
\hline 2.2 & -107.25168271 & 307744 & -107.2 & 538116 & -107.253 & 971 \\
\hline 2.4 & -107.23320836 & 499619 & -107.2 & -107.07751670 & -107.2 & -10 \\
\hline 2.6 & -107.21614706 & -107.07675501 & -107.21876928 & 929962 & -107.21 & -10 \\
\hline 2.8 & -107.20079141 & 49123 & -107.20372282 & 523 & -107.2 & -107.0 \\
\hline 3 & -107.18713289 & -107.0802 & -107.19021872 & -107.08224322 & -107.1 & -107.0 \\
\hline 3.2 & 1707 & -107.081 & -107.17809937 & 244 & -107. & -107. \\
\hline 3.4 & 25490 & -107 & -10 & 49 & & \\
\hline 3.6 & -107.15465471 & -107.08477098 & & 81 & 21 & 117 \\
\hline 3.8 & -107.14606377 & -107.08599834 & & & -107.15085481 & -107.0896632 \\
\hline 4 & -107.13833885 & -107.08704638 & -107 & 218 & -107.14322615 & -107.0905960 \\
\hline 4.2 & -107.13137779 & -107.08793229 & -107.13322578 & 2214 & -107.13634105 & -107. \\
\hline 4.4 & -107. & -107. & -107.1 & -10 & -10 & \\
\hline 4.6 & & & -107.1 & -107.0 & & \\
\hline 4.8 & 10 & & -107.1 & -107 . & & \\
\hline 5 & -107.10951336 & -107 & -107.1 & $-10^{\prime}$ & 54723 & -107.09255250 \\
\hline 5.2 & -107.10524990 & -107.09030650 & & & -107.10996936 & -107.0927507 \\
\hline 5.4 & -107.10141369 & -107.09038969 & & & -107.10604616 & -107.0928770 \\
\hline 5.6 & -107.09805973 & -107.09024801 & -10 & & -107.10228531 & -107.0928818 \\
\hline 5.8 & 378 & 332 & -107. & 80 & -107.0 & $95^{\circ}$ \\
\hline 6 & -10 & -10 & -107.09431200 & 8350 & -107.0 & -10 \\
\hline 6.2 & 303404 & -107 & -107.09336841 & -107.08583938 & -107.09510996 & -107.0908037 \\
\hline 6.4 & -107.09274666 & -107.08371373 & -107.09293606 & -107.08357200 & -107.09453854 & -107.0886739 \\
\hline 6.6 & -107.09262192 & -107.08131938 & -107.09273168 & -107.08124257 & -107.09432411 & -107.086351 \\
\hline 6.8 & -107.09256314 & -107.07900366 & & & -107.09422959 & -107.0840588 \\
\hline 7 & -107.09253530 & -107.07679314 & -107.09257535 & -107.07676860 & -107.09418200 & \\
\hline 7.2 & -107.09252397 & -107.07469433 & -107.09254887 & -107.07468000 & -107.09415600 & -107.0798911 \\
\hline
\end{tabular}

${ }^{1}$ Bao J. J.; Zhou, C.; Varga, Z.; Kanchanakungwankul, S.; Gagliardi, L.; Truhlar, D. G. Multi-state pairdensity functional theory. Faraday Discuss. 2020, online as Article ASAP. doi.org/10.1039/D0FD00037J. 


$\begin{array}{ccccccc}7.4 & -107.09251991 & -107.07270208 & -107.09253559 & -107.07269358 & -107.09414098 & -107.07789903 \\ 7.6 & -107.09251874 & -107.07080973 & -107.09252869 & -107.07080463 & -107.09413177 & -107.07669432 \\ 7.8 & -107.09251946 & -107.06901268 & -107.09252582 & -107.06900959 & -107.09412508 & -107.07489923 \\ 8 & -107.09252171 & -107.06730610 & -107.09252579 & -107.06730423 & -107.09412068 & -107.07319312\end{array}$

Table S2. Absolute CMS-PDFT energies (hartrees) for $\mathrm{LiH}$

\begin{tabular}{ccccc} 
& CMS- & CMS- & CMS- & CMS- \\
$R($ Li-H) & PDFT_1 & PDFT_2 & PDFT_3 & PDFT_4 \\
1 & -7.95794844 & -7.80934844 & -7.72138428 & -7.70653256 \\
1.2 & -8.02032697 & -7.86982169 & -7.78516455 & -7.76978622 \\
1.4 & -8.04515734 & -7.90065472 & -7.81672168 & -7.80166941 \\
1.6 & -8.05094148 & -7.91641648 & -7.83170852 & -7.81741344 \\
1.8 & -8.04718741 & -7.92447703 & -7.83820188 & -7.82481869 \\
2 & -8.03888853 & -7.92859783 & -7.84042035 & -7.82809769 \\
2.2 & -8.02873091 & -7.93079245 & -7.84067286 & -7.82984653 \\
2.4 & -8.01816723 & -7.93207014 & -7.84025061 & -7.83195402 \\
2.6 & -8.00798484 & -7.9329125 & -7.840414 & -7.8349217 \\
2.8 & -8.00074964 & -7.93286181 & -7.84321612 & -7.83537294 \\
3 & -7.99230998 & -7.93133018 & -7.84675847 & -7.83460691 \\
3.2 & -7.98437955 & -7.92996204 & -7.85011471 & -7.83385929 \\
3.4 & -7.97774449 & -7.92824042 & -7.85374229 & -7.8332967 \\
3.6 & -7.97254754 & -7.92605461 & -7.85736058 & -7.83290833 \\
3.8 & -7.96867448 & -7.92340066 & -7.86074945 & -7.83267707 \\
4 & -7.96590951 & -7.92036205 & -7.86378143 & -7.83256593 \\
4.2 & -7.96399863 & -7.91708377 & -7.86639385 & -7.8325458 \\
4.4 & -7.96272199 & -7.91373647 & -7.86857778 & -7.83260465 \\
4.6 & -7.96187598 & -7.91044768 & -7.87031663 & -7.83270432 \\
4.8 & -7.96131002 & -7.90732167 & -7.87160669 & -7.83282395 \\
5 & -7.96093818 & -7.90445519 & -7.87246241 & -7.83296193 \\
5.2 & -7.96070191 & -7.90191976 & -7.87289481 & -7.83311509 \\
5.4 & -7.96054599 & -7.89974598 & -7.87289909 & -7.83327076 \\
5.6 & -7.96043398 & -7.89794875 & -7.87249364 & -7.83340671 \\
5.8 & -7.96035278 & -7.89652009 & -7.87172245 & -7.83354736 \\
6 & -7.9602964 & -7.89542813 & -7.87064858 & -7.83367777 \\
6.2 & -7.96025749 & -7.89461745 & -7.86934279 & -7.83380515 \\
6.4 & -7.96021174 & -7.89400902 & -7.86785716 & -7.83391081 \\
6.6 & -7.9601707 & -7.89356279 & -7.86625896 & -7.83399464 \\
6.8 & -7.96016623 & -7.89326828 & -7.86460901 & -7.83406827 \\
7 & -7.96021661 & -7.8931134 & -7.86294235 & -7.83413416 \\
7.2 & -7.96032155 & -7.89307611 & -7.86128297 & -7.83419179 \\
7.4 & -7.96045937 & -7.8931173 & -7.85964697 & -7.83424168 \\
7.6 & -7.96059064 & -7.89318369 & -7.858036 & -7.83427482 \\
8.8 & -7.96069592 & -7.89324561 & -7.85645977 & -7.8342931 \\
& -7.9607785 & -7.89329955 & -7.85492892 & -7.83430034 \\
\hline .2 & -7.96085136 & -7.89335413 & -7.8534481 & -7.83429692 \\
\hline & & & &
\end{tabular}




$\begin{array}{ccccc}8.4 & -7.96092632 & -7.89341808 & -7.85201957 & -7.83428649 \\ 8.6 & -7.96100478 & -7.89349045 & -7.8506445 & -7.83427129 \\ 8.8 & -7.96107852 & -7.89356129 & -7.84932419 & -7.8342516 \\ 9 & -7.96113248 & -7.89361427 & -7.84805696 & -7.83422688 \\ 9.2 & -7.96115086 & -7.89363256 & -7.84683609 & -7.83419716 \\ 9.4 & -7.96113733 & -7.89361923 & -7.84566226 & -7.83416753 \\ 9.6 & -7.96111213 & -7.89359434 & -7.84453751 & -7.83414118 \\ 9.8 & -7.96109876 & -7.89358152 & -7.84346137 & -7.83411845 \\ 10 & -7.96111281 & -7.89359665 & -7.84243258 & -7.83409523 \\ 10.2 & -7.96115606 & -7.89364142 & -7.84145039 & -7.83406926 \\ 10.4 & -7.96121648 & -7.89370317 & -7.84051521 & -7.83404275 \\ 10.6 & -7.96127461 & -7.89376211 & -7.83962847 & -7.83401208 \\ 10.8 & -7.96131177 & -7.89379973 & -7.8387933 & -7.83396838 \\ 11 & -7.96131766 & -7.89380583 & -7.83801717 & -7.83390551 \\ 11.2 & -7.96128931 & -7.89377732 & -7.83731015 & -7.83381284 \\ 11.4 & -7.96123299 & -7.8937203 & -7.83668499 & -7.83367141 \\ 11.6 & -7.96116376 & -7.89364975 & -7.83617535 & -7.83346722 \\ 11.8 & -7.96109961 & -7.89358372 & -7.83577485 & -7.8331563 \\ 12 & -7.96105396 & -7.89353572 & -7.83552995 & -7.83281137 \\ 12.2 & -7.9610348 & -7.89351422 & -7.83537094 & -7.83234525 \\ 12.4 & -7.96103814 & -7.89351577 & -7.83530225 & -7.83195894 \\ 12.6 & -7.96105759 & -7.89353357 & -7.83525911 & -7.83144077 \\ 12.8 & -7.96107775 & -7.89355306 & -7.83525323 & -7.8311567 \\ 13 & -7.96109214 & -7.89356627 & -7.83524319 & -7.83065667\end{array}$

Table S3. Absolute CMS-PDFT energies (hartrees) for phenol

\begin{tabular}{cccccc}
\multicolumn{3}{c}{ dihedral =1 deg } & \multicolumn{3}{c}{ dihedral =10 deg } \\
$R(\mathrm{O}-\mathrm{H})$ & CMS-PDFT_1 & CMS-PDFT_2 & $R(\mathrm{O}-\mathrm{H})$ & CMS-PDFT_1 & CMS-PDFT_2 \\
0.746945 & -306.9686557 & -306.7902951 & 0.5 & -306.2493353 & -306.0708683 \\
0.796945 & -307.0073071 & -306.8290081 & 0.55 & -306.5153003 & -306.336953 \\
0.846945 & -307.0310263 & -306.8527733 & 0.6 & -306.6990932 & -306.5208584 \\
0.896945 & -307.0440825 & -306.8658661 & 0.65 & -306.8256161 & -306.6474793 \\
0.946945 & -307.0495214 & -306.8713346 & 0.7 & -306.9118778 & -306.7338041 \\
0.996945 & -307.0495582 & -306.8713983 & 0.75 & -306.9712922 & -306.7928889 \\
1.046945 & -307.0458088 & -306.8676764 & 0.8 & -307.0089055 & -306.8305695 \\
1.096945 & -307.0394509 & -306.8613505 & 0.85 & -307.0318724 & -306.8535892 \\
1.146945 & -307.0313533 & -306.8532913 & 0.9 & -307.0443871 & -306.8661486 \\
1.196945 & -307.0221414 & -306.8441268 & 0.95 & -307.0494412 & -306.8712437 \\
1.246945 & -307.0122789 & -306.8343212 & 1 & -307.0492081 & -306.8710513 \\
1.296945 & -307.0021055 & -306.8242129 & 1.05 & -307.0452696 & -306.8671574 \\
1.346945 & -306.9918607 & -306.8140414 & 1.1 & -307.0387842 & -306.8607249 \\
1.396945 & -306.9827516 & -306.8493217 & 1.15 & -307.0306048 & -306.8526123 \\
1.446945 & -306.9729714 & -306.8528325 & 1.2 & -307.0213503 & -306.8434443 \\
1.496945 & -306.9635146 & -306.8562068 & 1.25 & -307.0114798 & -306.8336901
\end{tabular}




\begin{tabular}{|c|c|c|c|c|c|}
\hline 1.546945 & -306.9544477 & -306.8593739 & 1.3 & -307.0013475 & -306 \\
\hline 1.596945 & -306.9458275 & -306.8623018 & 1.35 & -306.9913887 & -306.8142143 \\
\hline 646945 & -306.9376886 & -306.8649787 & 1.4 & -306.9820058 & 4272 \\
\hline 696945 & -306.9300612 & -306.8674088 & 1.45 & -306.9722312 & -306.8528749 \\
\hline 746945 & -306.9229647 & -306.8696055 & 1.5 & -306.9627842 & -306 \\
\hline 1.796945 & -306.9164099 & -306.8715879 & 1.55 & -306.9537375 & -306.8593435 \\
\hline 1.846945 & -306.9104044 & -306.8733701 & 1.6 & -306.9451381 & -306.8622499 \\
\hline 1.896945 & -306.9049438 & -306.8749688 & 1.65 & -306.9370282 & -306.8649126 \\
\hline 1.946945 & -306.9000204 & -306.8764007 & 1.7 & -306.929432 & -306.8673308 \\
\hline 1.996945 & -306.8956127 & -306.8776831 & 1.75 & -306.9223749 & -306.8695172 \\
\hline 2.046945 & -306.8916994 & -306.8788295 & 1.8 & -306.9158712 & -306.8714869 \\
\hline 2.096945 & -306.8882535 & -306.8798523 & 1.85 & -306.9099206 & -306.8732521 \\
\hline 2.146945 & -306.8852412 & -306.880762 & 1.9 & -306.9045253 & -306.8748262 \\
\hline 2.196945 & -306.8826413 & -306.8815512 & 1.95 & -306.8996819 & -306.8762223 \\
\hline 2.246945 & -306.8823277 & -306.8803194 & 2 & -306.8953725 & -306.8774507 \\
\hline 2.296945 & -306.882964 & -306.8783759 & 2.05 & -306.8915872 & -306.878513 \\
\hline 2.346945 & -306.8835358 & 767091 & 2.1 & & -306.87939 \\
\hline 2.396945 & -306.8840455 & -306.87529 & 2.15 & -306.885668 & -306.8799952 \\
\hline 2.446945 & -306.8845003 & -306.8740825 & 2.2 & -306.8838799 & -306.8800145 \\
\hline 2.496945 & -306.8849061 & -306.8730608 & 2.25 & -306.8833463 & -306.8790416 \\
\hline 2.546945 & -306.8852663 & -306.8722007 & 2.3 & -306.8835419 & -306.8775776 \\
\hline 2.596945 & -306.8855853 & -306.8714796 & 2.35 & -306.883925 & -306.876136 \\
\hline 2.646945 & -306.885871 & -306.8708768 & 2.4 & -306.8843341 & -306.8748506 \\
\hline 2.696945 & -306.886126 & -306.8703748 & 2.45 & -306.884726 & -306.8737348 \\
\hline 2.746945 & -306.8863544 & -306.869957 & 2.5 & -306.885088 & -306.8727831 \\
\hline 2.796945 & -306.8865596 & -306.8696113 & 2.55 & -306.8854181 & -306.8719783 \\
\hline 2.846945 & -306.8867436 & -306.8693265 & 2.6 & -306.8857162 & -306.8713019 \\
\hline 2.896945 & -306.8869083 & -306.8690919 & 2.65 & -306.8859841 & -306.8707343 \\
\hline 2.946945 & -306.8870554 & -306.8689009 & 2.7 & -306.8862261 & -306.8702606 \\
\hline 2.996945 & -306.8871852 & -306.8687466 & 2.75 & -306.8864422 & -306.8698675 \\
\hline 3.046945 & -306.8873017 & -306.8686213 & 2.8 & -306.8866362 & -306.8695417 \\
\hline 3.096945 & -306.8874064 & -306.86852 & 2.85 & -306.8868112 & -306.8692721 \\
\hline 3.146945 & -306.8875006 & -306.868438 & 2.9 & -306.8869676 & -306.8690503 \\
\hline 3.196945 & -306.8875845 & -306.8683723 & 2.95 & -306.8871057 & -306.8688681 \\
\hline
\end{tabular}

Table S4. Absolute CMS-PDFT energies (hartree) for HNCO

$150 \mathrm{deg}$

$\begin{array}{ccccc}R(\mathrm{C}-\mathrm{N}) & \text { CMS-PDFT_1 } & \text { CMS-PDFT_2 } & \text { CMS-PDFT_1 } & \text { CMS-PDFT_2 } \\ 1.3 & -168.27381244 & -168.26203907 & -168.28417209 & -168.26117433 \\ 1.4 & -168.28544670 & -168.27715856 & -168.29442630 & -168.27776912 \\ 1.5 & -168.28503252 & -168.27984983 & -168.29256645 & -168.28092568 \\ 1.6 & -168.27907241 & -168.27552161 & -168.28505990 & -168.27682301 \\ 1.7 & -168.27142350 & -168.26808617 & -168.27575555 & -168.26939962\end{array}$




$\begin{array}{ccccc}1.8 & -168.26398972 & -168.26040861 & -168.26699926 & -168.26132122 \\ 1.9 & -168.25794089 & -168.25388331 & -168.25998362 & -168.25439353 \\ 2 & -168.25398232 & -168.24974550 & -168.25523578 & -168.24998426 \\ 2.1 & -168.25231935 & -168.24864816 & -168.25290493 & -168.24863905 \\ 2.2 & -168.25229376 & -168.24958394 & -168.25251046 & -168.24947599 \\ 2.3 & -168.25314377 & -168.25132305 & -168.25318911 & -168.25119849 \\ 2.4 & -168.25423480 & -168.25306538 & -168.25421054 & -168.25295647 \\ 2.5 & -168.25520607 & -168.25447436 & -168.25517907 & -168.25440352 \\ 2.6 & -168.25592062 & -168.25546608 & -168.25594308 & -168.25546759 \\ 2.7 & -168.25637385 & -168.25609927 & -168.25648536 & -168.25619694 \\ 2.8 & -168.25666283 & -168.25649912 & -168.25683657 & -168.25666719 \\ 2.9 & -168.25704083 & -168.25694660 & -168.25701426 & -168.25692163 \\ 3 & -168.25707942 & -168.25701977 & -168.25718290 & -168.25714001\end{array}$

Table S5. Absolute CMS-PDFT energies (hartrees) for methylamine

\begin{tabular}{|c|c|c|c|c|c|c|c|}
\hline \multirow[b]{2}{*}{$R(\mathrm{~N}-\mathrm{H})$} & \multicolumn{2}{|c|}{$90 \mathrm{deg}$} & \multicolumn{2}{|c|}{$100 \mathrm{deg}$} & \multicolumn{3}{|c|}{0 deg } \\
\hline & CMS-PDFT_1 & CMS-PDFT_2 & CMS-PDFT_1 & CMS-PDFT_2 & $\mathrm{R}(\mathrm{N}-\mathrm{H})$ & CMS-PDFT_1 & CMS-PDFT_2 \\
\hline 0.616 & -95.33867984 & -95.13391247 & -95.33761335 & -95.12500502 & 0.602 & -95.30222741 & -95.09564912 \\
\hline 0.716 & -95.54743362 & -95.34697169 & -95.54639834 & -95.33815342 & 0.702 & -95.5326518 & -95.32990009 \\
\hline 0.816 & -95.64697826 & -95.45126471 & -95.64597793 & -95.44254889 & 0.802 & -95.64405638 & -95.44532499 \\
\hline 0.916 & -95.68699913 & -95.49643428 & -95.68641899 & -95.4887937 & 0.902 & -95.69116489 & -95.4969825 \\
\hline 1.016 & -95.6966193 & -95.50931185 & -95.69468715 & -95.50482928 & 1.002 & -95.70330175 & -95.51482019 \\
\hline 1.116 & -95.68882809 & -95.51158075 & -95.68559625 & -95.50712312 & 1.102 & -95.69881011 & -95.51268254 \\
\hline 1.216 & -95.6728858 & -95.50995667 & -95.66743258 & -95.50549 & 1.202 & -95.6840129 & -95.51007383 \\
\hline 1.316 & -95.65001568 & -95.5014997 & -95.65078608 & -95.50535638 & 1.302 & -95.66481298 & -95.50895192 \\
\hline 1.416 & -95.62842547 & -95.50308631 & -95.62599601 & -95.49891635 & 1.402 & -95.64333341 & -95.50876417 \\
\hline 1.516 & -95.60599539 & -95.50739835 & -95.60428115 & -95.50334785 & 1.502 & -95.62100992 & -95.50744608 \\
\hline 1.616 & -95.58466481 & -95.51248525 & -95.58294672 & -95.50835454 & 1.602 & -95.59587821 & -95.50898501 \\
\hline 1.716 & -95.56542365 & -95.5170882 & -95.56380943 & -95.51283654 & 1.702 & -95.57715911 & -95.51325047 \\
\hline 1.816 & -95.54862055 & -95.52081255 & -95.54725434 & -95.51631396 & 1.802 & -95.56029301 & -95.51715858 \\
\hline 1.916 & -95.53471951 & -95.5232304 & -95.53396401 & -95.5181345 & 1.902 & -95.54541484 & -95.52057018 \\
\hline 2.016 & -95.52833928 & -95.51973997 & -95.52699869 & -95.51525357 & 2.002 & -95.53255079 & -95.52345341 \\
\hline 2.116 & -95.52914159 & -95.51066041 & -95.52648854 & -95.5075129 & 2.102 & -95.5258315 & -95.52167051 \\
\hline 2.216 & -95.53028044 & -95.50277681 & -95.52722239 & -95.50005618 & 2.202 & -95.52775001 & -95.51267713 \\
\hline 2.316 & -95.53122435 & -95.49647973 & -95.52799251 & -95.49395456 & 2.302 & -95.52927464 & -95.50540197 \\
\hline 2.416 & -95.5319812 & -95.49158289 & -95.52866152 & -95.4891608 & 2.402 & -95.53047423 & -95.49964552 \\
\hline 2.516 & -95.53258937 & -95.48784639 & -95.52920087 & -95.48550498 & 2.502 & -95.53140913 & -95.49519643 \\
\hline 2.616 & -95.5330879 & -95.48504439 & -95.52966603 & -95.48274459 & 2.602 & -95.53213235 & -95.49180714 \\
\hline 2.716 & -95.53350599 & -95.48297119 & -95.5300429 & -95.48071851 & 2.702 & -95.53269797 & -95.48927699 \\
\hline 2.816 & -95.53384751 & -95.48146866 & -95.53036797 & -95.47923499 & 2.802 & -95.53313887 & -95.48741864 \\
\hline 2.916 & -95.53413617 & -95.48038554 & -95.53063242 & -95.47818035 & 2.902 & -95.5334865 & -95.48607354 \\
\hline 3.016 & -95.53438125 & -95.47961206 & -95.53089448 & -95.47738824 & 3.002 & -95.5337642 & -95.48511119 \\
\hline 3.116 & -95.53458793 & -95.4790656 & -95.53108226 & -95.47686385 & 3.102 & -95.53398531 & -95.48443557 \\
\hline
\end{tabular}




$\begin{array}{llllllll}3.216 & -95.53476045 & -95.47868284 & -95.53124635 & -95.4764918 & 3.202 & -95.53416234 & -95.48396609 \\ 3.316 & -95.53490156 & -95.47841467 & -95.53138576 & -95.47622617 & 3.302 & -95.53430487 & -95.48364371 \\ 3.416 & -95.53501392 & -95.47823109 & -95.53149491 & -95.47604735 & 3.402 & -95.53441926 & -95.48342567 \\ 3.516 & -95.53510665 & -95.47810634 & -95.53159883 & -95.47591245 & 3.502 & -95.53451025 & -95.48328286 \\ 3.616 & -95.535183 & -95.47802283 & -95.53167375 & -95.47582963 & 3.602 & -95.53458293 & -95.48318933 \\ 4.116 & -95.53536976 & -95.47787371 & -95.53186506 & -95.47567348 & 4.602 & -95.53476491 & -95.48303817 \\ 5.116 & -95.53541344 & -95.47783273 & -95.53192372 & -95.4756202 & 5.602 & -95.53476399 & -95.48305288 \\ 7.116 & -95.53597499 & -95.47827047 & -95.53195354 & -95.47563173 & 7.602 & -95.53476067 & -95.48305559\end{array}$

Table S6. Absolute CMS-PDFT and XMS-PDFT energies (hartrees) for the spiro cation

\begin{tabular}{|c|c|c|c|c|}
\hline $\mathrm{RC}$ & CMS-PDFT_1 & CMS-PDFT_2 & XMS-PDFT_1 & XMS-PDFT_2 \\
\hline-1.5 & $-612.05101 \overline{36}$ & $-612.01448 \overline{38}$ & $-612.051364 \overline{2} 1$ & -612.01483044 \\
\hline-1.45 & -612.0531267 & -612.0178089 & -612.05350251 & -612.01818072 \\
\hline-1.4 & -612.0551416 & -612.0210368 & -612.05554542 & -612.02143649 \\
\hline-1.35 & -612.057061 & -612.0241667 & -612.05749592 & -612.02459735 \\
\hline-1.3 & -612.0588836 & -612.0271992 & -612.05935324 & -612.02766439 \\
\hline-1.25 & -612.0606109 & -612.0301356 & -612.06111936 & -612.03063955 \\
\hline-1.2 & -612.062243 & -612.0329756 & -612.06279531 & -612.03352320 \\
\hline-1.15 & -612.0637807 & -612.0357208 & -612.06438251 & -612.03631776 \\
\hline-1.1 & -612.065223 & -612.0383697 & -612.06588117 & -612.03902281 \\
\hline-1.05 & -612.0665728 & -612.0409252 & -612.06729546 & -612.04164260 \\
\hline-1 & -612.0678293 & -612.0433861 & -612.06862604 & -612.04417750 \\
\hline-0.95 & -612.0689929 & -612.045753 & -612.06987566 & -612.04663019 \\
\hline-0.9 & -612.0700646 & -612.0480268 & -612.07104773 & -612.04900409 \\
\hline-0.85 & -612.0710446 & -612.0502071 & -612.07214576 & -612.05130224 \\
\hline-0.8 & -612.0719331 & -612.0522942 & -612.07317465 & -612.05352945 \\
\hline-0.75 & -612.072731 & -612.0542886 & -612.07414083 & -612.05569194 \\
\hline-0.7 & -612.0734385 & -612.0561902 & -612.07505259 & -612.05779751 \\
\hline-0.65 & -612.0740561 & -612.057999 & -612.07592133 & -612.05985716 \\
\hline-0.6 & -612.0745849 & -612.0597154 & -612.07676343 & -612.06188645 \\
\hline-0.55 & -612.075025 & -612.0613385 & -612.07760094 & -612.06390673 \\
\hline-0.5 & -612.0753769 & -612.062869 & -612.07846790 & -612.06595195 \\
\hline-0.45 & -612.0756429 & -612.0643051 & -612.07941381 & -612.06806800 \\
\hline-0.4 & -612.0758235 & -612.0656459 & -612.08051716 & -612.07033167 \\
\hline-0.35 & -612.0759212 & -612.0668902 & -612.08190568 & -612.07286746 \\
\hline-0.3 & -612.0759389 & -612.0680347 & -612.08379305 & -612.07588343 \\
\hline-0.25 & -612.0758816 & -612.0690751 & -612.08655619 & -612.07974814 \\
\hline-0.2 & -612.075759 & -612.0700012 & -612.09084068 & -612.08509088 \\
\hline-0.15 & -612.0755837 & -612.0708007 & -612.09784885 & -612.09309375 \\
\hline-0.1 & -612.0753864 & -612.0714448 & -612.10912822 & -612.10525592 \\
\hline-0.05 & -612.0752184 & -612.0718823 & -612.12408544 & -612.12088892 \\
\hline 0 & -612.0751491 & -612.0720408 & -612.13260086 & -612.12967700 \\
\hline 0.05 & -612.0752184 & -612.0718823 & -612.12408544 & -612.12088892 \\
\hline 0.1 & -612.0753864 & -612.0714448 & -612.10912822 & -612.10525592 \\
\hline 0.15 & -612.0755837 & -612.0708007 & -612.09784885 & -612.09309375 \\
\hline 0.2 & -612.075759 & -612.0700012 & -612.09084068 & -612.08509088 \\
\hline
\end{tabular}




$\begin{array}{ccccc}0.25 & -612.0758816 & -612.0690751 & -612.08655619 & -612.07974814 \\ 0.3 & -612.0759389 & -612.0680347 & -612.08379305 & -612.07588343 \\ 0.35 & -612.0759212 & -612.0668902 & -612.08190568 & -612.07286746 \\ 0.4 & -612.0758235 & -612.0656459 & -612.08051716 & -612.07033167 \\ 0.45 & -612.0756429 & -612.0643051 & -612.07941381 & -612.06806800 \\ 0.5 & -612.0753769 & -612.062869 & -612.07846790 & -612.06595195 \\ 0.55 & -612.075025 & -612.0613385 & -612.07760094 & -612.06390673 \\ 0.6 & -612.0745849 & -612.0597154 & -612.07676343 & -612.06188645 \\ 0.65 & -612.0740561 & -612.057999 & -612.07592133 & -612.05985716 \\ 0.7 & -612.0734385 & -612.0561902 & -612.07505259 & -612.05779751 \\ 0.75 & -612.072731 & -612.0542886 & -612.07414083 & -612.05569194 \\ 0.8 & -612.0719331 & -612.0522942 & -612.07317465 & -612.05352945 \\ 0.85 & -612.0710446 & -612.0502071 & -612.07214576 & -612.05130224 \\ 0.9 & -612.0700646 & -612.0480268 & -612.07104773 & -612.04900409 \\ 0.95 & -612.0689929 & -612.045753 & -612.06987566 & -612.04663019 \\ 1 & -612.0678293 & -612.0433861 & -612.06862604 & -612.04417750 \\ 1.05 & -612.0665728 & -612.0409252 & -612.06729546 & -612.04164260 \\ 1.1 & -612.065223 & -612.0383697 & -612.06588117 & -612.03902281 \\ 1.15 & -612.0637807 & -612.0357208 & -612.06438251 & -612.03631776 \\ 1.2 & -612.062243 & -612.0329756 & -612.06279531 & -612.03352320 \\ 1.25 & -612.0606109 & -612.0301356 & -612.06111936 & -612.03063955 \\ 1.3 & -612.0588836 & -612.0271992 & -612.05935324 & -612.02766439 \\ 1.35 & -612.057061 & -612.0241667 & -612.05749592 & -612.02459735 \\ 1.4 & -612.0551416 & -612.0210368 & -612.05554542 & -612.02143649 \\ 1.45 & -612.0531267 & -612.0178089 & -612.05350251 & -612.01818072 \\ 1.5 & -612.0510136 & -612.0144838 & -612.05136421 & -612.01483044\end{array}$

